\title{
Der Kulturlandschaftswandel in der Schweiz als geographisches Forschungsprogramm
}

\section{Vielgestaltige Schweiz - auch in Zukunft?}

Fragen wir nach dem Ausgangspunkt für das Studienprojekt «Kulturlandschaftswandel» («KLW»), so ist er in der allgemeinen Besorgnis zu finden, die gegenwärtigen Veränderungen könnten die vielgestaltige Schweiz zu einem technogenen, kaum mehr differenzierten Lebensraum werden lassen. $\mathrm{Da}$ diese Sorge um eine kulturräumlich anregende Umwelt berechtigt ist, erhärtet der Augenschein in unseren wachsenden Städten, aber auch überall dort, wo in ländlichen Gebieten die Bautätigkeit eingesetzt hat. Es sei dabei nicht die Meinung vertreten, $\mathrm{da} ß$ die Landschaft in alle Zukunft etwas Ruhendes bleiben müsse; gerade bei einer vom Menschen bestimmten "Kulturlandschaft» ist Veränderung, Umbau, Anpassung an neue Lebensbedingungen unausweichlich. Jedoch sollte man sich über die räumlichen Konsequenzen in der eingeschlagenen Richtung der Veränderung im Klaren sein; man muß bedenken, daß vielleicht gleichzeitig an vielen Orten unseres Landes in freiem Entschluß dieselbe Richtung der Veränderung gewählt wird, so daß die Gefahr der (vielleicht ungewollten) Konvergenz der Raumgestaltung besteht. Es muß verhindert werden, $\mathrm{da} ß$ unser unteilbarer Lebensraum durch eine unbekümmerte «Nutzungsmentalität» gewissermaßen «Stück um Stück» verbraucht wird. Wenn an irgendeiner Stelle unseres Landes eine ausgedehnte Großverteileranlage errichtet wird, so ist an dieser Stelle wie auch in deren Umgebung jede andere Nutzung meist versperrt. Korrekturen etwaiger Fehlanlagen lassen sich nachträglich nur mit kaum verantwortbaren Aufwendungen durchsetzen. Wenn man die Prozesse der räumlichen Veränderung genau verfolgt, wie es in zahlreichen geographischen Analysen der Gegenwart geschieht, so ist man von der relativen Einförmigkeit der technischen Mittel der Umgestaltung beeindruckt: Ausbau des Straßennetzes, Überbauung des Agrarraumes nach dem Muster der «Flurbereinigung», Abbruch älterer Gebäude und Aufbau höherer Gebäude. Es entsteht dieselbe «vernünftige» Fluraufteilung hier wie dort, dieselben Straßenanlagen durchziehen die Waadt wie die Ostschweiz; die gleichen zweckmäßigen Hochbauten zieren die Tessiner Städte wie Bern, Zürich oder Zug. An so und sovielen Orten unseres Landes beklagt die mittlere und ältere Generation den Umbau des gewohnten Stadtbildes, derweil die Jungen die permanente Veränderung als «normal» empfinden mögen. Freies Feld ändert die Hand und wird zum Betonbezirk. Bergbahnen leiten die Erschließung einsamer Berggegenden ein, derweil landwirtschaftlich unrentabel gewordene Kulturlandareale als «Brachland» dem Allerweltsrezept «Aufforstung» unterliegen. Sowohl Aufforstung als auch touristische Erschließung eröffnen nach unseren Erfahrungen den Homogenisierungsproze $\beta$ des Lebensraumes. Beide Maßnahmen haben ihren Sinn, wenn sie im Rahmen eines gesamträumlichen Konzepts eingeleitet werden; ihre Gefahr besteht in der allgemeinen und bedenkenlosen Handhabung. Zudem muß ausgesprochen werden, daß im jetzigen Zeitpunkt von der sich erst entwickelnden Schweizer Raumplanung noch keine korrigierende und harmonisierende Wirkung ausgehen kann. Damit erscheint der baldige Einsatz des KLW-Grundlagenprogrammes, eines Mittels der zuverlässigen Information, als besonders dringlich. Im Rahmen dieser Ausführungen ist der Ausgangsort für das KLW-Programm klar umrissen: Es soll den permanenten Umbau unseres Lebensraumes in seinen verschiedenen Erscheinungsformen bewußt werden lassen und durch diese Erkenntnis dazu beizutragen, daß unserer Bevölkerung die anregende Vielfalt des schweizerischen Lebensraumes auch in $\mathrm{Zu}$ kunft erhalten bleibt.

\section{Grundsätze des KLW-Forschungsprojektes}

Im Anschluß an die Ausarbeitung einer sozialgeographischen Analyse einer Tessiner Kulturlandschaft, insbesondere aber nach Abschluß der aktualgeographischen Studie über ländliche Räume der Nordwestschweiz reifte der Entschluß, die ganze Schweiz einer ähnlichen Analyse zu unterziehen. Die problematische Umgestaltung des Landes sollte Ziel einer speziellen geographischen Untersuchung werden, die sich im wesentlichen nach folgenden sieben Grundsätzen ausrichtete.

Prof. Dr. W. Gallusser, Geogr. Institut der Universität Basel, Klingelbergstraße 16, 4000 Basel 


\subsection{Komplexität der Raumdynamik}

Die räumliche Dynamik soll möglichst in ihrer gesamten Vielschichtigkeit erfaßt werden. Es geht also weniger darum, allein die Auswirkungen des Autobahnbaus über die ganze Schweiz hin zu untersuchen, als vielmehr den Autobahnbau neben andern raumverändernden Prozessen zu erfassen und ihn als eine raumdynamische Komponente zu den andern in Beziehung zu setzen.

\subsection{Regionale Streuung von Testräumen}

Um möglichst verschiedenartige raumdynamische Varianten fassen zu können, muß die Untersuchung auf möglichst unterschiedlich strukturierte Regionen ausgerichtet werden. Somit repräsentiert die Auswahl geeigneter Testräume bis zu einem gewissen Grad das Spektrum des Wandels im ganzen Bereich der Schweiz.

\subsection{Gleiche Methodik für die ganze Schweiz}

Im Gegensatz zu bisherigen Untersuchungen, die in kleineren, geschlossenen Gebieten den Strukturwandel analysierten und im einzelnen wohl interessante aber mit anderen Räumen kaum vergleichbare Resultate erbracht haben, soll in unserem Testnetz die gleiche Methodik für ein großes $\mathrm{Ge}-$ biet - natürlich nach dem Case-study-Verfahren zur Anwendung kommen. Damit wird für das ganze Land eine direkte Vergleichbarkeit der Resultate erreicht.

Die methodische Basis für das KLW-Programm bilden die Kartierungen, wie sie in Kapitel 5 genauer dargelegt werden. Es handelt sich dabei um die detaillierten Aufnahmen der kulturräumlichen Strukturen (d. h. der Bevölkerung, der Nutzflächen, der Gebäude), der funktionalen Zusammenhänge (der betrieblichen und sozialen Organisationen) sowie der für die Raumdynamik maßgebenden Grundbesitzverhältnisse. Die Kartierungsergebnisse sollten in geeigneter Form zusammengefaßt und publiziert werden. Liegt nach einem bestimmten zeitlichen Abstand (z. B. nach 10 Jahren) eine neue Serie von Kartierungsergebnissen vor, so wird es damit möglich, den Kulturlandschaftswandel zwischen den beiden Kartierungsphasen in seinem wirklichen Ausmaß aufzuzeigen und zu begründen.

\subsection{Permanente Untersuchung}

So wie eine räumlich schlüssige Untersuchung die Berücksichtigung der strukturell verschiedenartigen Teilräume verlangt (Punkt 2.2.), so ist eine den Zeitfaktor wesentlich einbeziehende Studie langfristig anzulegen. Am ehesten wäre - analog zur Beobachtung der Gletscherbewegung in der Schweiz - eine unbefristete permanente Untersuchung vorzusehen. Sofern die unbefristete Beobachtung und Auswertung gewährleistet wäre, ließe sich das Ausmaß der Veränderungen mit größerer Sicherheit beurteilen, indem sich die nachhaltige und langfristige von der zeitlich und räumlich belanglosen Dynamik klar abheben würde.

\subsection{Regelmäßige Untersuchungsserien}

Aus methodischen und praktischen Gründen müssen die einzelnen Untersuchungsschritte auf bestimmte Jahre verteilt werden. Analog den Volkszählungen wären die Teiluntersuchungen in regelmäßig wiederkehrenden Erhebungs- und Auswertungsphasen anzuordnen. Dabei drängt sich eine zeitliche Zuordnung zu den entsprechenden Terminen der Schweizer Statistik auf, so daß z. B. die Siedlungsanalyse mit dem Volkszählungsjahr oder die Nutzungsanalyse eher mit dem Betriebszählungsjahr koordiniert werden könnte. Auf jeden Fall sollten die gleichen Untersuchungen in den Testgebieten in regelmäßigen Abständen wiederholt werden. Nach den Erfahrungen der Schweizer Statistik erscheint der 10 Jahres-Rhythmus auch für unser Programm vertretbar.

\subsection{Interkantonaler Forschungsstab}

Wenn die zeitliche Abfolge der Teiluntersuchungen genügend Raum für die Auswertung und für die voraussichtliche Publikation der Befunde läßt, ist das Arbeitsprogramm zumindest im Gelände nur durch einen interkantonalen Forschungsstab zu bewältigen. Die Gleichzeitigkeit der einzelnen Untersuchungsschritte (Punkt 2.5.) und die regionale Streuung (Punkt 2.2.) zwingen zur parallel durchgeführten Analyse. Weil das Programm nach Punkt 2.4. langfristig angelegt werden soll, ist die Arbeitsweise auch insofern vorteilhaft, als sich die regionalen Mitarbeiter mit den besonderen Strukturen 
und der Problematik ihres Testgebietes intensiver auseinandersetzen können. Ein gebietsfremder Bearbeiter, der zudem meist nur während einer einzigen Untersuchungskampagne tätig ist, könnte diese «regionale Zuständigkeit» nicht gewährleisten.

\subsection{Optimale Koordination}

Die regionale Streuung der Mitarbeiter erfordert eine wirksame Zusammenfassung, Betreuung und Auswertung der einzelnen Arbeitsschritte. Diese Arbeit ist vorläufig am ehesten an einem bestehenden geographischen Hochschulinstitut zu leisten, und zwar durch einen ausgebildeten Geographen, der sich vollberuflich der optimalen Koordination und der Auswertung der Ergebnisse widmen könnte. Gerade in der Anfangsphase, aber auch später, wären Tagungen für den Forschungsstab vorzubereiten, an denen die Methodik einläßlich diskutiert und instruiert werden müßte.

\section{Der Werdegang des KLW-Projektes}

\subsection{Die grundsätzliche Motivierung}

Wie schon vermerkt, gehört die Untersuchung räumlicher Veränderungen zu den klassisch zu nennenden Aufgaben der Geographie. Gesamthaft gesehen wurden aber in der Fragestellung eher die langfristig verlaufenden Veränderungen, d.h. die säkularen Vorgänge, in den humangeographischen Arbeiten berücksichtigt. Meist ergaben sich die Einblicke durch den Vergleich von Landschaftsquerschnitten, die in großen Zeitabständen, nach Maßgabe der vorhandenen Quellen, rekonstruiert werden konnten. Aus den meist nur dürftigen historischen Grundlagen sind die wirklichen, vielschichtigen Prozesse über Jahrzehnte und Jahrhunderte hinweg nur vereinfacht, lückenhaft und zusammenfassend erschließbar. Gegenüber der Breite der Gegenwartsanalyse verengert sich der Aussageumfang des historisch-geographischen Rückgriffes: Der vom kontinuierlichen Wandlungsproze $B$ erfaßte Raum würde eine viel dichter angelegte Retrospektive erforderlich machen, um den heutigen $\mathrm{Zu}$ stand aus dem historischen Wandel heraus voll be- greiflich zu machen. Wieviel Wesentliches für die heutige Raumproblematik wäre etwa noch von einer intensiven Erforschung der einsetzenden Industrialisierung Europas zu erwarten? Jedenfalls übersteigt allein schon eine solche Fragestellung die Möglichkeiten der Geographie, sofern sich nicht die Geschichte in verstärktem Maße dem Thema zuwendet. Zweifellos vermittelt die Kenntnis des langfristigen Wandels, gewonnen durch die historisch-geographische Betrachtungsweise, auch für die Beurteilung der heutigen und der zukünftigen Raumsituation eine klärende Hilfe. Die retrospektive Untersuchung wird weiterhin gepflegt werden müssen, und zwar mit Vorteil in enger Tuchfühlung mit der historischen Disziplin. Aus den eingangs dargelegten Gründen verlangt jedoch der beschleunigte, zunehmend folgenschwerere Wandel des gegenwärtigen Lebensraumes nach einer permanenten wissenschaftlichen Untersuchung. Sofern heute die Gewichte der geographischen Forschung neu verteilt werden müßten, wäre der Abklärung der aktuellen Raumdynamik vermehrtes Gewicht, ja Vorrang einzuräumen. Ist noch 1928 die «Dynamische Länderkunde» eines SPETHMANN ein interessanter Versuch ohne nachhaltige Wirkung geblieben, so fordert heute das zeitgenössische Umwelt-Bewußtsein die Geographie zur wissenschaftlichen Abklärung der aktuellen Raumdynamik, reportierend und analysierend im Schritte der Zeit geradezu heraus.

\subsection{Die Startphase 1969-1970}

Wie schon ausgeführt, bestand 1969 - im Anschluß an die aktual-geographische Untersuchung derNordwestschweiz durch W. Gallusser - die Absicht, die ganze Schweiz im Case-study-Verfahren geographisch zu analysieren. Allerdings mußte dazu erst eine tragfähige Grundlage in personeller und finanzieller Hinsicht gefunden werden. Dies geschah an der Sitzung der Forschungskommission des "Verbandes Schweiz. Geogr. Gesellschaften" (später «Schweiz. Geogr. Gesellschaft») am 6. Sept. 1969 in Olten. PD Dr. W. Gallusser wurde als neugewähltes Mitglied der Forschungskommission beauftragt, den Kulturlandschaftswandel in der Schweiz mit Unterstützung des geographischen Dachverbandes als gesamtschweizerisches Forschungsprojekt vorzubereiten. Mit der Neustrukturierung des geographi- 
Abb. 1: Ayent VS mit Ferienzentrum Anzère; gemischte

Erwerbsstruktur, Mischsiedlung im Berggebiet.

(Photo: Schweizerische Verkehrszentrale, Zürich)

Abb. 2: Sempach LU; gemischte Erwerbsstruktur, Mischsiedlung auBerhalb Berggebiet.

(Photo: Schweizerische Verkehrszentrale, Zürich)
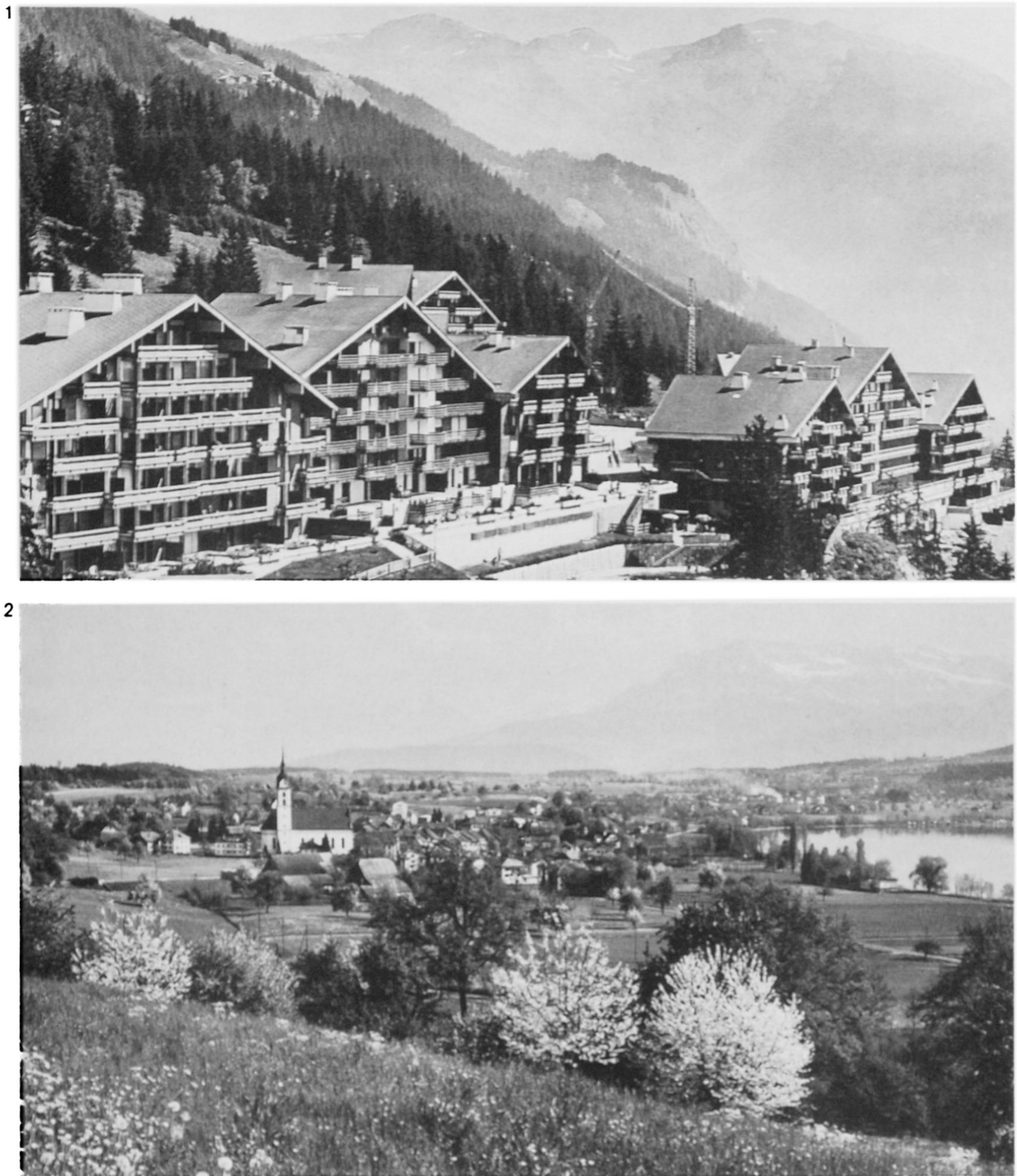
Abb. 3: Ennenda GL; Industriegemeinde, Mischsiedlung

im Berggebiet. (Photo: Swissair-Photo AG, Zürich)

Abb. 4: Kaisten AG; gemischte Erwerbsstruktur, Kernsied-

lung außerhalb Berggebiet. (Photo: J. Probst)
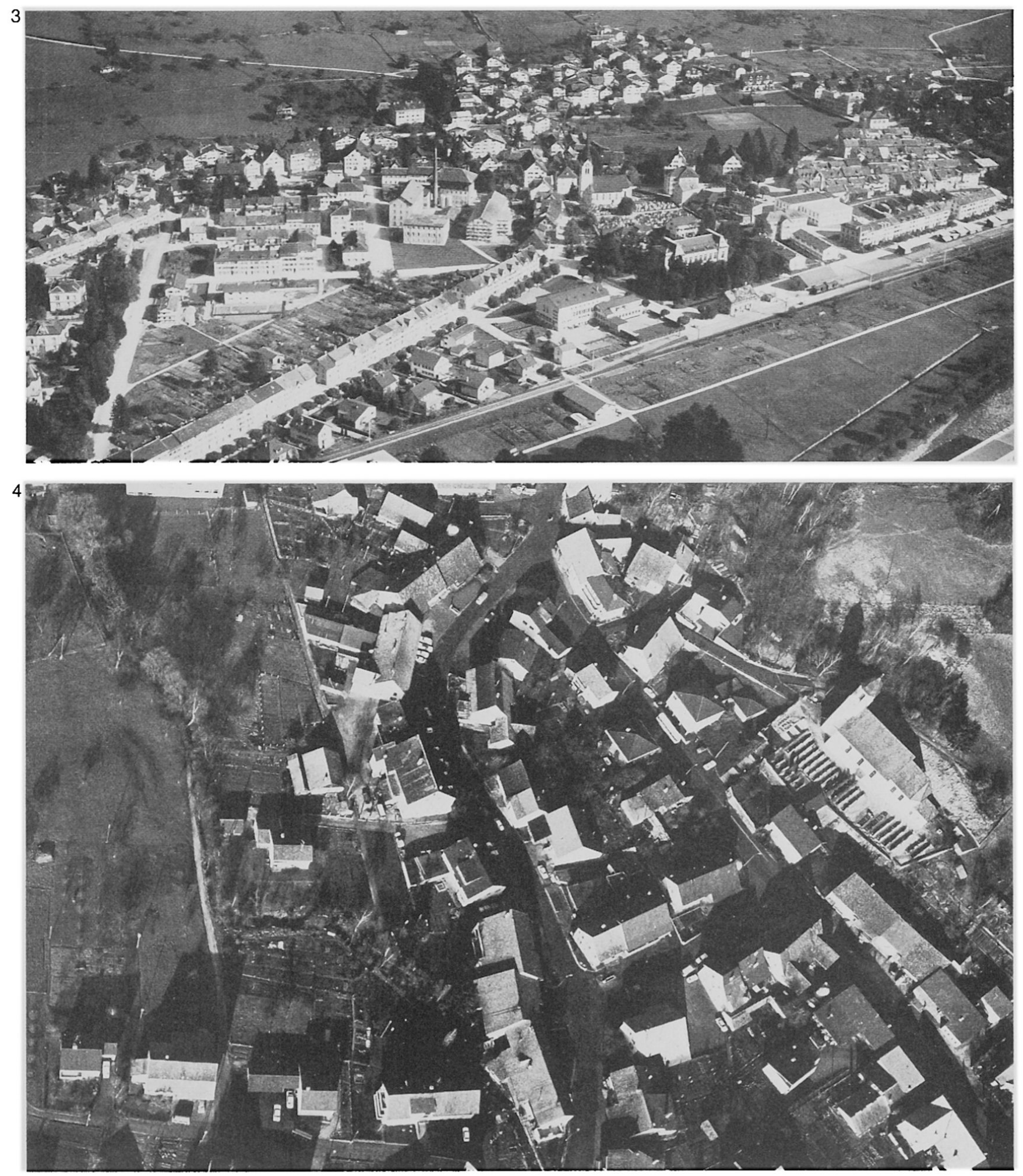
schen Dachverbandes zur "Schweizerischen Geographischen Gesellschaft» und der gleichzeitigen Gründung einer «Schweizerischen Geographischen Kommission» (SGgK) durch die Schweiz. Naturforschende Gesellschaft (SNG, Mai 1970) konnten die organisatorischen Möglichkeiten auch für das KLW-Vorhaben verbessert werden. Es ist der Tatkraft der Kollegen Dr.E. Bugmann (Präsident der Schweiz. Geogr. Ges.) und Prof. Dr. G. Grosjean (Präsident der SGgK) zu danken, daß dem Projekt die gerade am Anfang nötige finanzielle und moralische Unterstützung zuteil wurde, vor allem auch von Seiten der SNG. Während des Sommersemesters 1970 beschäftigte sich eine Arbeitsgemeinschaft des Geogr. Institutes der Universität Basel eingehend mit den Prinzipien des KLW-Projektes. Dabei schälte sich u. a. die Erkenntnis heraus, daß die Auswahl der Testgemeinden im Blick auf die regionale und die strukturelle Vielfalt mit dem Computer erfolgen müsse.

\subsection{Das Vorprojekt 1970-1971}

Vorerst galt es nun, eine kleinere Studiengruppe zu formieren, und zwar mit folgender Zusammensetzung:

PD Dr. K. Aerni, Bern

Dr. F. Chiffelle, Neuenburg

Dr. U. Eichenberger, Basel-Stadt (Bis 1971)

PD Dr. H. Elsasser, Zürich

Prof. Dr. W. Gallusser, Basel-Stadt, SG (Präsident)

Dr. E. Schwabe, Bern (Kassier)

Die beiden letztgenannten sind Mitglieder der SGgK. Deren Präsident (Prof. Dr. G. Grosjean) gehört der Studiengruppe ex officio an. 1970-1971 fanden acht Sitzungen in Basel, Bern, Kaisten/AG, Olten, Muri/ BE und Vinelz/BE statt. Man beschloß, das KLWProjekt vorerst in einem "Vorprojekt» zu testen und die Methodik ausgiebig in größeren Fachgremien diskutieren zu lassen. Während dieser Projektphase sollte auch die Auswahl der Testgemeinden und der Aufbau des Mitarbeiterstabes erfolgen. Zu Beginn wurden die Methoden in zwei grundsätzlich voneinander abweichenden Raumtypen, nämlich in industrialisierten bzw. in agrarischen Testgebieten erprobt und für das Hauptprojekt bereinigt. Ein erstes Studienteam wählte dazu die ländliche $\mathrm{Ge}$ meinde Vinelz/BE und wurde dabei durch Berner Geographiestudenten von Prof. Grosjean unterstützt. Die Gemeinde Kaisten/AG diente als Testraum des neu industrialisierten Gebietes Sisselnfeld-Laufenburg; die entsprechenden Geländeuntersuchungen wurden im April 1971 im Rahmen offizieller Feldstudien von Studenten des Geogr. Institutes der Universität Basel durchgeführt. Am 12. Dez. 1970, anläßlich des ersten Schweizer Geographiesymposions der SGgK in Bern, wurde die Idee des KLW-Projektes und die ersten Erfahrungen mit dem Vorprojekt einem größeren Fachpublikum vorgestellt. Das positive Echo bestärkte die Studiengruppe in ihrer Zielsetzung. Aufgrund der vorliegenden Feldversuche wurden im Frühjahr 1971 die Kriterien für die Auswahl der Testgemeinden festgelegt, worauf man das Programm für den Basler Universitätscomputer vorbereitete. Zusammen mit dem ORL-Institut der ETHZ (H. Elsasser) und der Abteilung für Angewandte Geographie der Universität Bern (G. Grosjean) koordinierte das Geograph. Institut der Universität Basel (W. Gallusser) die Computerarbeit, so daß man im Sommer 1971 die Testgemeinden anhand der vorliegenden Plotterkarten endgültig auswählen konnte. Nachdem die Zahl von 24 Testgemeinden feststand, mußte der Mitarbeiterstab zusammengestellt werden. Ein Aufruf in Heft 3 der "Geographica Helvetica» 1971 sowie persönliche Umfragen waren erfolgreich, so daß auf Ende 1971 die Zusammensetzung des Bearbeiterteams provisorisch feststand. Im Rahmen des 2. Symposions der SGgK in Basel (12. Febr. 1972) konnten die bisherigen Ergebnisse des KLW-Vorprojektes durch die Studiengruppe wiederum einem größeren Fachgremium zur Diskussion vorgelegt werden, und zwar wie folgt:

\footnotetext{
- Grundsätze und Methodik des KLW-Programmes (Gallusser)

- Das Auswahlverfahren für die Testgemeinden

(Elsasser)

- Die Testgemeinden und der Mitarbeiterstab (Schwabe)

- Die Betriebsbefragung am Beispiel einer Neuenburger Gemeinde (Chiffelle)

- Die Grundbesitzkartierung am Beispiel einer

Berner Gemeinde (Aerni)
} 
- Die Nutzflächenkartierung am Beispiel der Gemeinde Vinelz (Grosjean)

\subsection{Das KLW-Programm 1972-1974}

Nach dem Ablauf des Vorprojektes galt es, das Hauptprogramm in zwei Arbeitsphasen anlaufen zu lassen. Die Phasierung war einesteils durch die vorläufig beschränkte Finanzierung über den Schweizer Nationalfonds, andernteils aus personellen Gründen bedingt. Zudem erwies es sich als vorteilhaft, 19721973 eine erste Arbeitsphase in einem Teil der Testgemeinden vorzuschalten, damit man vor Beginn der zweiten Arbeitsphase (ab 1973) in den übrigen Testgemeinden noch von einem letzten Erfahrungsaustausch profitieren konnte. Die Studiengruppe koordinierte 1972-1973 an vier Sitzungen in Basel und Olten die fortlaufenden Arbeiten. Dazu wurden zwei Mitarbeiter-Tagungen organisiert: am 22. April 1972 fand in Olten eine gutbesuchte Konferenz statt, wo man das Programm und die Methodik der ersten Arbeitsphase (1972-1973) festlegte. An einer anderthalbtägigen Konferenz in St. Gallen (28.-29. April 1973) versammelten sich 22 Mitarbeiter zur Erledigung folgender Haupttraktanden:

1. Erfahrungsaustausch betr. erste Arbeitsphase (1972-1973)

2. Instruktion über die Kartierungsmethodik

a) Nutzflächenkartierung (Elsasser)

b) Grundbesitzkartierung (Amrein)

c) Gebäudekartierung (Grosjean)

3. Aufgaben des KLW-Programms 1974-1975 (Gallusser)

Die St. Galler Arbeitskonferenz leitete die zweite Arbeitsphase ein, welche voraussichtlich Mitte 1974 - im Zeitpunkt der Publikation dieser Zeilen - abgeschlossen werden kann. Es ist vorgesehen, die Ergebnisse des KLW-Programmes 1972-1974 in zweckdienlicher Form zu veröffentlichen.

\section{Die Testgemeinden und ihre Bearbeiter (Abb. 1-5)}

Ohne in Einzelheiten auf das Auswahlverfahren einzugehen, sei vorerst das Ausscheidungsprinzip erläutert. In einem ersten Durchgang wurden drei kodierte Hauptmerkmale berücksichtigt, wobei die methodischen Erfahrungen von GUTERSOHN (1950) sowie von BOBEK, HAMMER und OFNER (1955) dienlich waren. Die Hauptmerkmale sollen den Kulturraum in den wichtigsten Zügen charakterisieren. Dazu eignen sich u. E. die Erwerbsstruktur (landwirtschaftliche, industrielle, Dienstleistungs- und gemischte Gemeinden), die Siedlungsweise (Kern-, Misch- und Streusiedlung) sowie die Lage in bezug auf die landwirtschaftliche Berggrenze (Berggebiet, übriges Gebiet). Von den 3095 Gemeinden wurden 24 der 6 häufigsten Kombinationstypen auf den Plottern nach regionalen Gesichtspunkten ausgewählt. In einem zweiten Durchgang prüfte man die erste Auswahl nach den drei Nebenmerkmalen «Bahnverkehrslage» (Gemeinde mit oder ohne Bahnstation), "Autobahnlage» (Gemeinde von der Autobahn berührt oder nicht) und «Touristische Erschließung» (Fremdenverkehrsgemeinden; übrige). Entsprechend deren Häufigkeitsverteilung wurden wenn nötig Anpassungen vorgenommen. Ein letzter Durchgang korrigierte die Auswahl der 24 Testgemeinden nochmals nach der regionalen Repräsen$\operatorname{tanz}$ und der praktischen Eignung. Karte 1 gibt die räumliche Verteilung der Testgebiete wieder. Die Abb. 2-5 mögen einen gewissen Eindruck von der strukturellen und dynamischen Vielfalt der Testgemeinden vermitteln.

Weil die Untersuchungsgebiete über die Schweiz zerstreut liegen, war es wichtig, wenn möglich einheimische oder mit den lokalen Verhältnissen vertraute Mitarbeiter zu gewinnen. Mit wenigen Ausnahmen ist uns dies auch gelungen, wie dies aus der nachstehenden Zusammenstellung hervorgeht.

\begin{tabular}{ll}
\hline Testgemeinde & Bearbeiter (mit Wohnort) \\
\hline Hombrechtikon ZH & $\begin{array}{l}\text { Dr. P. Abt, Hombrechtikon } \\
\text { PD Dr. H. Elsasser, Zürich }\end{array}$ \\
Blauen BE & Prof. Dr. W. Gallusser, Basel \\
Gelterfingen BE & $\begin{array}{l}\text { PD Dr. K. Aerni, } \\
\end{array}$ \\
Kirchlindach BE & Premgarten b. Bern \\
& Prof. Dr. G. Grosjean, \\
Vinelz BE & Dr. R. Amrein, Bern \\
Sempach LU & Dr. R. Martin, Luzern \\
& Frau Dr. U.Zwahlen, Sempach \\
Seedorf UR & M. Rothenfluh, Altdorf
\end{tabular}


Fig. 1: Die KLW-Testgemeinden.

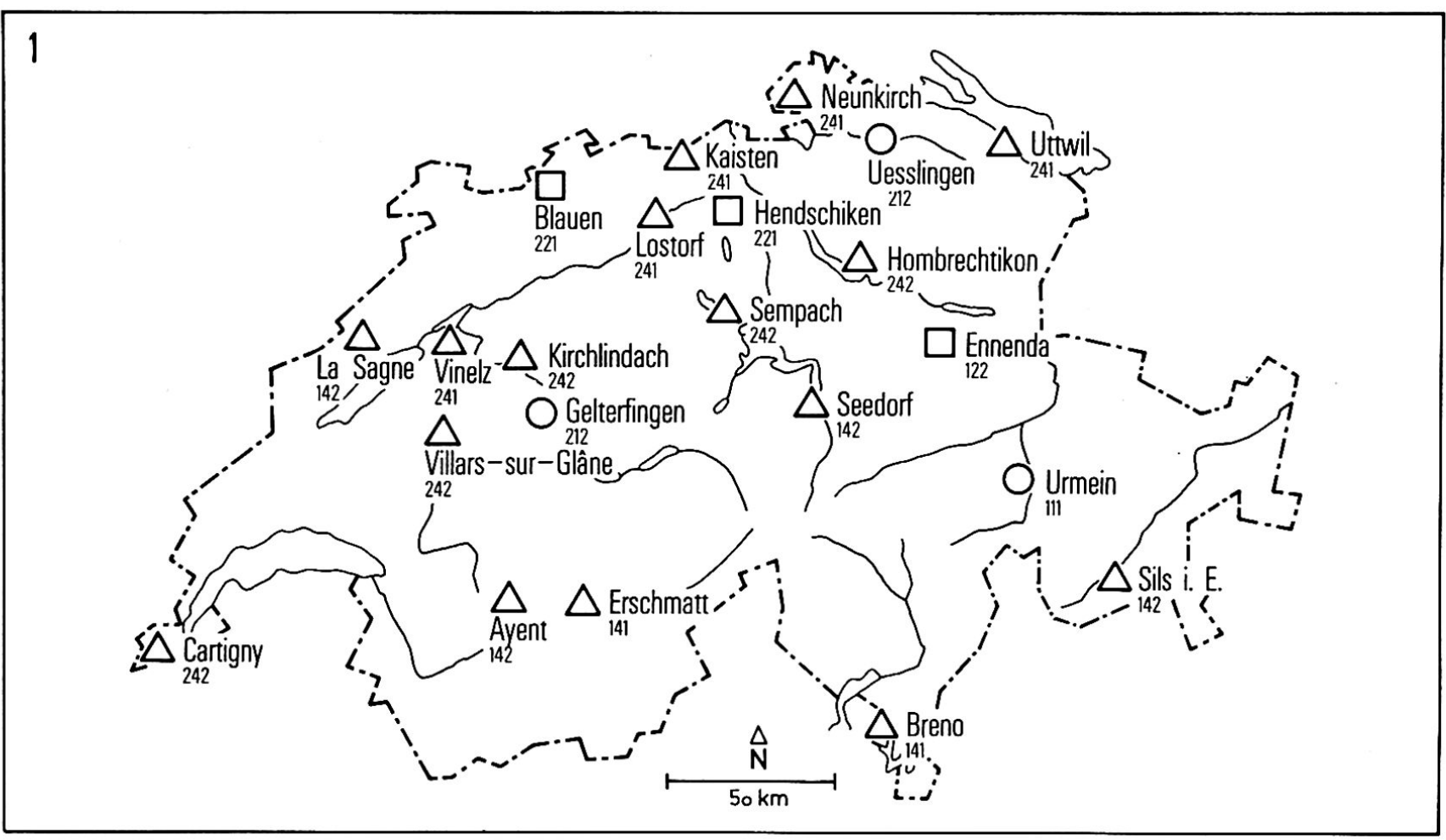

Ennenda GL Villars-sur-Glâne FR

H. Gasenzer, Glarus

Prof. Dr. J.-L. Piveteau, Villars-sur-Glâne U. Schnydrig, Fribourg

Lostorf SO

Neunkirch SH

Sils i. E. GR

Urmein GR

$\begin{array}{ll}\text { Hendschiken AG } & \text { Dr. R. Meier, Ennetbaden } \\ \text { Kaisten AG } & \text { Dr. A. May, Langenthal } \\ \text { Uesslingen TG } & \text { Dr. H. Andresen, Frauenfeld } \\ \text { Uttwil TG } & \text { Prof. Dr. H. Frey, St. Gallen } \\ \text { Breno TI } & \text { Dr. W. Leimgruber, Basel } \\ \text { Ayent VS } & \text { Dr. E. Schwabe, Muri b. Bern } \\ \text { Erschmatt VS } & \text { Dr. G. Budmiger, Aarberg } \\ \text { La Sagne NE } & \text { Prof. Dr. F. Chiffelle, } \\ & \text { Neuchâtel } \\ \text { Cartigny GE } & \text { Prof. Dr. C. Raffestin, Genève }\end{array}$

Es ist vorgesehen, daß eine Testgemeinde je nach den gegebenen Umständen räumlicher (große Einwoh- nerzahl, große Fläche, komplizierte Siedlungsverhältnisse) oder personeller Art (beschränkte zeitliche Möglichkeiten des Bearbeiters) von mehreren Geographen gemeinsam oder im Rahmen von Praktikumsarbeiten (durch Geographiestudenten) bearbeitet werden kann. Jedoch muß für jede Testgemeinde ein Verantwortlicher bezeichnet werden, der die Kontinuität der Untersuchung gewährleistet. $\mathrm{Da}$ die Mitarbeit finanziell nicht eigentlich voll entschädigt werden kann, sondern nur auf der Basis der Spesendeckung und einer bescheidenen Arbeitstags-Entschädigung (nach den Ansätzen der SNG-Kommissionen) erfolgt, macht das bisher Geleistete unserer Mitarbeiter um so bemerkenswerter. Immerhin darf der wissenschaftliche und persönliche Gewinn, der sich aus der Mitwirkung an einem derartigen Gemeinschaftswerk ergibt, nicht zu gering veranschlagt werden. Neben den ehrenamtlich tätigen Mitgliedern der Studiengruppe hilft uns seit 1973 lic. phil. W. Buchmann als Assistent mit koordinierenden Funktionen. Er wurde durch die Hochschule St. Gallen als wissenschaftlicher Teilzeit-Assistent dem KLW-Programm bis Ende März 1974 zur Verfügung gestellt. 


\section{Das bisherige Kartierungsprogramm}

(Abb. 6-12)

\subsection{Die Kartierungsgrundlagen 1972-1974 (Nutzflä- chen-, Grundbesitz- und Gebäudeaufnahme)}

Gemäß Zeitplan sollen in der jetzt laufenden Arbeitsphase die Nutzflächen, der Grundbesitz und die Gebäude in den Testgemeinden kartiert werden. Die eine Hälfte der Mitarbeiter hat mit den Aufnahmen schon 1972 begonnen, die andern setzten im Sommer 1973 oder in der folgenden Zeit mit ihrer Arbeit ein. Es wäre das Ziel, bis zum Frühsommer 1974 alle laufenden Aufnahmen abzuschließen. Darauf soll u. a. die Betriebskartierung vorbereitet werden, die 1975 im Anschluß an die Eidg. Betriebszählung durchzuführen ist.

Heute besitzen alle Mitarbeiter Instruktionen zur Aufnahme der Nutzflächen-, Grundbesitz- und Gebäudekarten. Es wurde vorgesehen, die Nutzflächen und den Grundbesitz im Maßstab 1:5000 zu kartieren, die Gebäude, soweit in geschlossener Siedlung, im Maßstab $1: 2000$. Wünschbar wäre es, wenn jeder Mitarbeiter von seiner Testgemeinde Tochterpausen in diesen zwei Maßstäben hätte; eine Umfrage an der Tagung in St. Gallen hat gezeigt, daß dieser Wunsch für 10 Testgemeinden, mindestens teilweise, bereits erfüllt werden konnte.

Zur Nutzflächenkartierung und zur Grundbesitzkartierung wurde von der KLW-Studiengruppe je eine Legende ausgearbeitet. Beide Legenden verwenden Flächenfarben und z.T. zusätzliche Raster, mit denen die entsprechenden Parzellen auf den Karten angegeben werden. Die Nutzflächenlegende dürfte für alle in der Schweiz vorkommenden Nutzungsarten genügen; bei lokalen Sonderformen kann sie noch von Fall zu Fall ergänzt werden.

Grundsätzlich sind alle Mitarbeiter angehalten, in der Legende festgehaltene Farben und Signaturen nicht anderweitig zu verwenden und nicht zu generalisieren, da sonst die Vergleichbarkeit zwischen den Testgemeinden beeinträchtigt würde. Mit zur Aufnahme gehört auch eine Aufstellung über die Flächenanteile; diese Angaben werden durch Planimetrieren der fertigen Karten gewonnen oder können - für die Grundbesitzanteile - in vielen Fällen den Grundbucheintragungen entnommen werden.
Über die heute geltenden Legenden zur Nutzflächen-, Grundbesitz- und Gebäudekartierung orientiert der nachfolgende Abschnitt. Die Abbildungen 6-12 geben von den Kartierungen aus verschiedenen Testgemeinden einige Probeausschnitte wieder. Sie sind als erste und unverbindliche graphische Umsetzungen der Kartierungsgrundlagen zu betrachten. Infolge der Schwarz-weiß-Reproduktion entsprechen die Abb. 6, 7 und 8 nicht der Originallegende; auch in den Abb. 9-12 mußte die Originallegende aus drucktechnischen Gründen leicht modifiziert werden.

\subsection{Die Kartierungslegenden}

Bei der Nutzflächenkartierung werden die Areale mit den entsprechenden Farben - allenfalls ergänzt durch einen Raster - bezeichnet. Ähnlich wird bei der Grundbesitzkartierung vorgegangen, wobei aber zur Grundfarbe, die die berufliche und juristische Stellung des Grundbesitzers bezeichnet, die Schraffur und Punktierung als Träger zusätzlicher Informationen hinzutritt.

Als Grundlage für die Gebäudekartierungen dienen Pläne, auf denen die Gebäude in Umrissen und nicht als ausgefüllte Flächen dargestellt sind. Die Signaturen werden in diese Gebäudeumrisse hineingezeichnet, entsprechend den Legenden zur Gebäudekartierung.

Die vollständigen Legenden sind diesem einleitenden Artikel angefügt.

\subsection{Der heutige Stand der $K L W$-Arbeit}

In zwei Regionen, in der Lavaux und im Sopra Ceneri, konnte aus personellen Gründen noch keine definitive Wahl einer Testgemeinde getroffen werden. In den meisten andern Testgebieten wurden jedoch die Aufnahmen begonnen und $\mathrm{z}$. T. bereits abgeschlossen. Am weitesten fortgeschritten sind die Nutzflächenkartierungen; die Grundbesitzaufnahmen befinden sich demgegenüber noch etwas im Rückstand (in drei Fällen müssen die fertigen Karten nochmals überarbeitet werden, um sie der revidierten Legende anzupassen). Von den aufwendigen Gebäudeaufnahmen, die aber unabhängig von der Jahreszeit durchgeführt werden können, liegen erwartungsgemäß erst wenige vor. 
Über den genauen Stand der Arbeiten in den einzelnen Testgemeinden gibt die folgende Tabelle Auskunft, die auf einer am Ende des vergangenen Jahres durchgeführten Umfrage basiert:

\section{Stand der Arbeiten in den Testgemeinden} (Januar 1974)

\begin{tabular}{|c|c|c|c|c|}
\hline Testgemeinde & 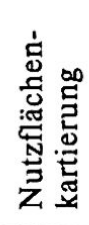 & 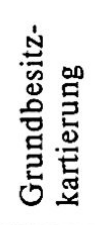 & 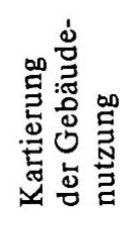 & 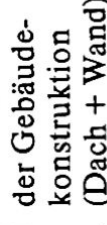 \\
\hline ZH Hombrechtikon & $\mathrm{O}$ & V & $\mathrm{O}$ & $\mathrm{O}$ \\
\hline BE Gelterfingen & $\mathrm{O}$ & V & 0 & O \\
\hline BE Kirchlindach & O & 0 & $x$ & $\mathrm{X}$ \\
\hline BE Vinelz & 0 & 0 & O & O \\
\hline LU Sempach & $\mathrm{O}$ & $\mathrm{O}$ & V & V \\
\hline UR Seedorf & V & V & $\mathrm{X}$ & $\mathrm{X}$ \\
\hline GL Ennenda & V & $\mathrm{O}$ & V & V \\
\hline FR Villars-sur-Glâne & 0 & V & $\mathrm{X}$ & $\mathrm{X}$ \\
\hline SO Lostorf & $\mathrm{O}$ & V & V & V \\
\hline GR Sils i. E. & $x$ & V & V & V \\
\hline GR Urmein & ○ & 0 & O & $X$ \\
\hline AG Hendschiken & $\mathrm{O}$ & V & V & V \\
\hline AG Kaisten & $\mathrm{O}$ & V & $\mathrm{O}$ & $\mathrm{X}$ \\
\hline TG Uesslingen & V & $\mathrm{X}$ & $\mathrm{X}$ & $\mathrm{X}$ \\
\hline TG Uttwil & $\mathrm{O}$ & $\mathrm{O}$ & V & $\mathrm{X}$ \\
\hline TI Breno & $\mathrm{O}$ & V & V & V \\
\hline VS Ayent & O & V & V & V \\
\hline VS Erschmatt & $\mathrm{O}$ & V & $\mathrm{X}$ & $\mathrm{X}$ \\
\hline NE La Sagne & 0 & 0 & 0 & 0 \\
\hline GE Cartigny & $x$ & V & V & V \\
\hline
\end{tabular}

$$
\text { Legende: } \begin{aligned}
\quad & =\text { vollständig fertig } \\
\mathrm{O} & =\text { Fast/zum Teil fertig (z. B. Karte ge- } \\
& \text { zeichnet, aber noch nicht planimetriert) } \\
\mathrm{V}= & \text { in Arbeit } \\
\mathrm{X}= & \text { noch nicht begonnen }
\end{aligned}
$$

\section{Ausblick}

Im jetzigen Zeitpunkt darf festgestellt werden, daß der Start des KLW-Programmes dank einer breiten Unterstützung durch die Schweizer Geographen geglückt ist. Es ist damit zu rechnen, daß gegen Ende
1974 die ersten Ergebnisse im Sinne der KLW-Grundsätze publikationsreif vorliegen dürften. Es muß aber darauf verwiesen werden, daß die Weiterführung des Programms ab 1975, d. h. die Erfassung des jetzigen Kulturlandschaftswandels durch Folgeerhebungen, aus finanziellen Gründen noch nicht gesichert ist.

Nach einer zweimaligen Subventionierung über den Schweizer Nationalfonds (1972-1974), welche als Überbrückungshilfe gewährt worden war, muß eine finanzielle Trägerschaft im Rahmen der SNG gefunden werden. Weil das KLW-Programm durch seine gesamtschweizerische Konzeption seit Anfang von der SGgK gefördert werden konnte, erscheint uns der Augenblick gekommen, das Forschungsvorhaben mit Hilfe der SNG wirksam und auf längere Zeit sicherzustellen. Eine Effizienz kann nach unseren bisherigen Erfahrungen aber nur erreicht werden, wenn es möglich wird, die weiträumig angelegten Untersuchungen durch eine einsatzfähige Koordinationsstelle zu unterstützen. Dabei ist es von der Sache her belanglos, wo dieses Koordinationszentrum lokalisiert und welche fachlich ausgewiesene Persönlichkeit mit dieser Funktion betraut werden wird. Heute ist es nur wichtig, daß diese Stelle die bisher von der Hochschule St. Gallen in Form einer Teilassistenz getragen wurde - voll in das KLW-Programm integriert wird. Kann diese wichtige Voraussetzung in Zukunft erfüllt werden, so ist damit zu rechnen, daß die Schweizer Geographen die Zielsetzung des KLW-Programmes erreichen können. Damit würde das Projekt über das rein fachwissenschaftlich Geographische hinaus eine allgemeinere Bedeutung für unser Land erlangen, wie wir das schon am ersten Symposion der SGgK 1970 zum Ausdruck gebracht haben.

Zugegeben, das KLW-Untersuchungsprogramm wird noch viele Fragen der Dynamik unseres Lebensraumes offenlassen. Nach wie vor wird das Hauptgewicht der Regionalforschung von den geographischen Instituten getragen werden müssen. Aber die folgenschwere Veränderung unserer Umwelt verlangt, gerade im Zeichen der sich anbahnenden nationalen Raumplanung, nach einer gesamtschweizerischen geographischen Klärung. Man sagt dem Schweizer Sinn für das Nüchterne, das Fundierte und das Notwendige nach. Hier - getragen von der SGgK der Schweizerischen Naturforschen- 
den Gesellschaft - werden sich Geographen, ungeachtet ihrer regionalen Gebundenheit, zu einem wissenschaftlichen und gleichwohl praktischen Werk zusammenfinden, welches von den Fundamenten der landschaftlichen Gegenwart ausgeht. Solcherart bietet die Erforschung des kulturlandschaftlichen Wandels eine geographische Orientierungshilfe auf dem Weg zur Schweiz von morgen.

\section{Résumé}

Le point de départ du projet d'études «transformation du paysage humanisé» («Kulturlandschaftswandel», KLW) est dû à l'appréhension, que les changements actuels pourraient amener la Suisse multiforme à un espace de vie technogène, à peine différencié. Ce souci d'un espace de vie diversifié, stimulateur géographique, se justifie par la vue de nos villes croissantes, mais également par l'activité de construction qui se développe dans les régions rurales. Il ne s'agit pas de se dire, que le paysage doit rester une fois pour toute quelquechose de stable, mais l'on devrait pouvoir voir clairement les conséquences spatiales des modifications que l'on a envisagées. Si l'on suit de près les processus des modifications spatiales, comme cela se fait actuellement dans de nombreuses analyses géographiques, l'on est frappé par l'uniformité relative des moyens techniques employés pour la transformation: extension du réseau routier, construction sur des surfaces vouées préalablement à l'agriculture, remembrement rural, démolission de bâtiments vétustes et édification de bâtiments plus élevés... et dans toute la Suisse l'on rencontre les mêmes répartitions parcellaires et les mêmes constructions routières. Le programme KLW doit nous rendre conscient de la transformation permanente de notre espace de vie - ceci dans toutes ses formes - et de ce fait contribuer à ce que la stimulante diversité de l'espace de vie suisse puisse être également à l'avenir conservée à la population. C'est dans ce but que des recherches géographiques spéciales sont effectuées, ceci dans l'ensemble d'après les sept principes suivants:

1. Complexité de la dynamique spatiale: il ne faut pas prendre en considération les seuls aspects de la dynamique, mais avant tout sa complexité.
2. Répartition régionale des communes-témoins: des régions aussi différentes que possible doivent être choisies.

3. Méthodologie universelle pour toute la Suisse: un réseau de communes-témoins doit être analysé selon les mêmes critères. Comme base on se servira de relevés du paysage humanisé (démontrant la structure de la population, des surfaces utilisables, des bâtiments, ainsi que les rapports fonctionnels - organisations des entreprises - et les rapports de la propriété foncière).

4. Recherche permanente: pour obtenir des résultats concluants, l'étude doit être faite à long terme, comparable à l'observation des déplacements des glaciers.

5. Séries de recherches régulières: les recherches doivent être effectuées à des époques bien définies, p. ex. à la suite du recensement fédéral de la population resp. du recensement fédéral des entreprises; un rythme de 10 années nous paraît également justifiable pour le programme KLW.

6. Equipe de recherche intercantonale: la dispersion régionale (point 2) et la réalisation simultanée des analyses (point 5) nous oblige à former une équipe intercantonale de collaborateurs. En raison du long terme des projets (point 4) la préférence est à donner à des collaborateurs régionaux, qui sont familiarisés avec leur région. En ce qui concerne la Suisse Romande, il serait particulièrement important que nous puissions trouver des géographes pour la zone-témoin Lavaux (canton de Vaud).

7. Coordination efficace: cette dispersion des collaborateurs exige une centralisation, un soin et une analyse efficaces. Ceci pourra être réalisé avec le plus de chances dans un institut géographique, où un géographe qualifié s'occupera à temps entier de ces tâches.

Les premiers pas concrets menant à la réalisation du projet KLW furent faits en 1969. Depuis 1970 les travaux sont en cours dans le cadre de la Commission Suisse de Géographie de la Société Helvétique des Sciences Naturelles, financièrement soutenus par le Fonds National Suisse pour un temps défini (1972-1974).

Après un projet préalable, après que les communestémoins aient été choisies et que les collaborateurs aient pu être désignés, la confection des cartes put 
être amorcée en 1972 dans une partie des communestémoins (cartes des surfaces utilisables, de la propriété foncière et de l'habitat); depuis 1973 les travaux sont en cours dans presque toutes les régions. Lors de plusieurs conférences les collaborateurs furent réunis afin de discuter de la marche à suivre et d'échanger leurs expériences réciproques.

A l'heure actuelle le lancement du programme KLW paraît avoir réussi grâce à l'appui massif des géographes suisses. Il faut souligner cependant que la continuation du Programme à partir de 1975 n'est pas encore assurée pour des raisons financières; le financement doit être effectué dans le cadre de la SHSN. Une efficacité ne peut être atteinte que si les recherches entreprises dans un vaste domaine peuvent trouver l'appui d'un poste de coordination capable. Si cette importante condition peut être remplie, il est à prévoir que les géographes suisses puissent atteindre le but fixé par le programme KLW. De ce fait, outre une réussite scientifique et géographique, le projet aurait une signification plus vaste pour notre pays, et ceci particulièrement à l'époque où l'aménagement national du territoire prend forme.

La recherche de la transformation du paysage humanisé peut ainsi offrir une aide d'orientation géographique pour la Suisse de demain.

(Traduit par C. Lamparter)

\section{Literaturhinweise}

BOBEK H., HAMMER A., OFNER R.: Beiträge zur Ermittlung von Gemeindetypen. Klagenfurt 1955.

BUDMIGER G.: Erschmatt (VS); Beitrag zur Siedlungsund Wirtschaftsgeographie der inneralpinen Zone. Bern 1970.

Die Geographische Kommission der SNG, ihr Forschungsprogramm: In "Geographica Helvetica», 26. Jg., H. 2, S. 72-80. Bern 1971.

GALLUSSER W.: Siedlungsentwicklung und Grundbesitzverhältnisse in der modernen Tessiner Kurlandschaft. In «Regio Basiliensis», Jg. 9, H. 1, S. 215 bis 235. Basel 1968.

GALLUSSER W.: Struktur und Entwicklung ländlicher Räume der Nordwestschweiz. Aktualgeographische Analyse der Kulturlandschaft im Zeitraum 1955 bis 1968. Basel 1970.

KNÖPFLI R.: Die Nachführung der Landeskarte der Schweiz. Internationales Jahrbuch für Kartographie, S. 168-173, Gütersloh 1970.

SPETHMANN H.: Dynamische Länderkunde. Breslau 1928. 